\title{
19 HLA-G A PUTATIVE SUSCEPTIBILITY GENE IN SCLERODERMA, BUT ONLY IN WOMEN
}

Doua F Azzouz, ${ }^{1}$ Julie Di Cristofaro, ${ }^{2}$ Sami B Kanaan, ${ }^{1}$ Jean Cabane, ${ }^{3}$ Dominique Farge, ${ }^{4,5}$ Yannick Allanore, ${ }^{6}$ Eric Hachulla, ${ }^{7}$ Jean Robert Harlé, ${ }^{8}$ Brigitte Granel, ${ }^{9}$ Marielle Martin, ${ }^{1}$ Jean Roudier, ${ }^{1}{ }^{10}$ Christophe Picard, ${ }^{2}$ Nathalie Lambert ${ }^{1}{ }^{1}$ INSERM UMR639, Marseille, France; '2Laboratoire d'Immunogénétique EFS Alpes Méditerranée, France; ${ }^{3}$ Service de Médecine Interne, Hôpital St Antoine, Paris, France; ${ }^{4}$ Service de Médecine Interne et Pathologie Vasculaire, Hôpital St Louis, Paris, France; ${ }^{5}$ INSERM U697, Hôpital St Louis, Paris, France; ${ }^{6}$ Université Paris Descartes, Service de Rhumatologie A, Hôpital Cochin, APHP \& INSERM U781, Hôpital Necker, Paris, France; ${ }^{7}$ Service de Médecine Interne, Centre National de Référence de la Sclérodermie Systémique, Hôpital Claude Huriez, Lille, France; ${ }^{8}$ Service de Médecine Interne, Hôpital La Conception, Marseille; ${ }^{9}$ Service de Médecine Interne, Hôpital Nord, Marseille, France; ${ }^{10}$ Service de Rhumatologie, Hôpital Ste Marguerite, Marseille, France

DFA and JDC equally contributed to the work.

10.1136/annrheumdis-2011-201236.19

Background Systemic sclerosis or Scleroderma (SSc) is an autoimmune disease affecting mostly women with a higher incidence between 40 and 60 years old. Several HLA class II genes have been associated with SSc depending on the clinical subset of the disease, limited cutaneous SSc (Ic-SSc) or diffuse cutaneous SSc (dc-SSc) or on specific autoantibodies, anticentromere antibodies (ACA) or antitopoisomerase (ATA) antibodies, respectively hallmarks of the above clinical subsets. HLA-G is a non classical HLA molecule with tolerance functions that may play a role in inflammatory diseases. A 14 bp sequence insertion/ deletion polymorphism (rs16375) in the $3^{\prime}$-untranslated region of the HLA-G gene has been associated to levels of soluble HLA-G (sHLA-G). The insertion is associated with lower levels.

Objectives In the current study, the authors propose to analyse this polymorphism in patients with SSc compared to healthy individuals. The authors furthermore stratified patients according to sex, clinical subsets and autoantibody status.

Patients and methods The authors genotyped 145 patients with SSc and 95 healthy controls by a previously developed two step multiplex SNaPshot method several HLA-G Single Nucleotide polymorphisms and the -14bp Ins/Del polymorphism.

Results Patients with SSc tend to have higher frequency of ins/ ins $(29 / 145,20.0 \%)$ genotypes compared with healthy controls $(11 / 95,11.6 \%, p=0.008)$. The frequency of Ins alleles compared to Del alleles is significantly increased among patients with SSc $(p=0.024)$. This association reveals interesting patterns when patients are divided by sex (women, $\mathrm{N}=110$ and men, $\mathrm{N}=35$ ). Men had similar frequencies of Del/Del, Del/Ins, Ins/Ins genotypes than healthy controls (respectively, $45.7 \%, 42.9 \%, 11.4 \%$ and $50.5 \%, 37.9 \%, 11.6 \%$ ), whereas women had different proportions of genotypes with Del/Del: $36.4 \%$, Del/Ins: $40.9 \%$ and Ins/Ins: $22.7 \%$. The difference in genotype repartition was statistically significant in women with SSc compared with healthy controls $(p=0.047)$ and the difference in allele frequency even stronger (Ins: $43.1 \%$ compared with $30.5 \%, \mathrm{p}=0.008$ ). 
Apart from the gender association an association with dcSSc with the Ins allele $(p=0.004)$, and a marginal association with lcSSc $(p=0.08)$ were also observed. Interestingly both autoantibody subgroups, ATA and ACA, were associated with higher Ins allele frequencies ( $p=0.018$ and $p=0.03$, respectively).

Conclusion Women with SSc but not men have a genotype of low sHLA-G secretor. Further studies need to be conducted to better understand this gender dichotomy and its meaning in tolerance in a disease affecting mostly women in their childbearing years. 